\title{
Minimizing distortion and enlarging group delay in Brillouin slow light systems by gain profile optimization
}

\author{
Liang Zhang ${ }^{1,2}$, Marcelo A. Soto ${ }^{1}$, Luc Thévenaz ${ }^{1}$ \\ ${ }^{1}$ EPFL Swiss Federal Institute of Technology, Institute of Electrical Engineering, \\ SCI STI LT, Station 11, CH-1015 Lausanne, Switzerland; \\ ${ }^{2}$ Department of Physics and Astronomy, Key Laboratory for Laser Plasmas (Ministry of Education), State Key Lab of Advanced Optical \\ Communication Systems and Networks, Shanghai Jiao Tong University, Shanghai, 200240, China; \\ E-mail: liangzai@sjtu.edu.cn,marcelo.soto@epfl.ch, luc.thevenaz@epfl.ch
}

\begin{abstract}
Optimal gain spectra minimizing distortion and enhancing delaying efficiency in linear slow light systems are predicted and experimentally validated by shaping the gain spectral profile in a Brillouin fiber amplifier.

OCIS codes: (060.4370) Nonlinear optics, fibers; (290.5900) Scattering, stimulated Brillouin; (350.5500) Propagation
\end{abstract}

\section{Introduction}

Controlling the speed of light in optical media has turned into one of the most promising fields during the past few years owing to its interesting potential applications as all-optical variable delay lines, light-matter interaction enhancement and signal processing [1]. Several slow light approaches have been demonstrated through the generation of high normal dispersion in sharp spectral resonances based on different physical interactions [1]. However, a limit to the maximum time delay always exists since amplitude and phase distortions can never mutually compensate in linear slow light systems and large delays are inevitably subject to major distortion [2].

In this paper, a strategy to minimize pulse distortion for a given delay is proposed and experimentally validated in a stimulated Brillouin scattering (SBS) slow light system. Considering a real limited gain bandwidth, a specific parabolic gain profile with symmetric sharp cutting edges turns out to be optimal for minimizing pulse distortion and enhancing the delay efficiency, rather than the flat-top gain profile, particularly for large fractional delays.

\section{Theoretical background and simulation results}

Like any linear slow light system, SBS-based slow light in optical fibers is inherently affected by distortion [1-3], resulting from the frequency-dependent Brillouin gain and nonlinear phase response. Compared to other methods [1], SBS offers interesting advantages, such as the possibility to arbitrary change the bandwidth and spectral shape of the gain medium [3,4], giving some freedom to minimize distortion and maximize delay efficiency. Unfortunately, these two conditions are widely incompatible, leading to the known tradeoff between delay and distortion [2].

In general, the root-mean-square (RMS) pulse width at the output of the system can be expressed as [2]:

$$
\sigma_{t}^{2}=\sigma_{X}^{2}+\sigma_{\varphi}^{2}=\frac{1}{E_{\omega}} \int_{-\infty}^{+\infty}\left|\frac{d X(\omega)}{d \omega}\right|^{2} d \omega+\frac{1}{E_{\omega}} \int_{-\infty}^{+\infty}|X(\omega)|^{2}\left|\frac{d \varphi(\omega)}{d \omega}\right|^{2} d \omega
$$

where the first $\left(\sigma_{x}\right)$ and second $\left(\sigma_{\varphi}\right)$ terms are the amplitude and phase contributions, respectively. $X(\omega)$ is the amplitude distribution and $\varphi(\omega)$ the phase distribution of the output pulse spectrum, which is the input pulse spectrum multiplied by the transfer function of the gain process. $E_{\omega}$ is the total energy of the pulse.

The slow light effect is predominantly driven by the limited bandwidth and the curvature of the gain profile, both being generically described by the simplified distribution $g(\omega)=\left[g_{0}-g_{2}(\omega / \Omega)^{2}+O\left(\omega^{4}\right)\right] \cdot \Pi(\omega /(\Omega / 2))$ for a gain window of strictly limited bandwidth $\Omega$. $g_{0}$ is the peak gain (in linear amplitude scale), $g_{2}$ is the gain profile index defining the spectral curvature, which takes positive values for slowing light effects (note the negative sign in front of $\left.g_{2}\right), \Pi(\cdot)$ is the rectangular function, and $O\left(\omega^{4}\right)$ represents higher-order terms which turn out to have a very moderate impact in practical cases. For a Gaussian-shaped input pulse, the amplitude, phase and total broadenings can be evaluated using Eq. (1) [2]. Fig. 1(a) shows the pulse broadening normalized to the input RMS pulse width $\left(\sigma_{i n}\right)$ as a function of the gain profile index $g_{2}$, for pulses of 880 ps delayed in a SBS slow light medium of $32 \mathrm{~dB}$ gain and $1 \mathrm{GHz}$ bandwidth. It can be observed that the amplitude distortion induced by the slow light system increases as $g_{2}$ increases from 0 to 1 , whilst the phase broadening exhibits a minimum at $g_{2}=0.5$. Note that, although no amplitude distortion $\left(\sigma_{X} / \sigma_{i n}=1\right)$ is expected for a flat-top gain profile $\left(g_{2}=0\right)$ [4], a significant broadening can still be obtained due to the dominant high levels of phase distortion $\left(\sigma_{\phi} / \sigma_{\text {in }}\right)$. On the other hand, when $g_{2}>\sim 0.4$, the contribution of the amplitude distortion turns out to be dominant. In consequence, a minimum total distortion can be found for an intermediate value of $g_{2}$, which in this case is close to 0.25 . 
Fig. 1(b) shows the linear relation between the index $g_{2}$ and the fractional delay (defined as the group delay $\phi_{1}$ divided by the input pulse width $\tau_{\text {in }}$ ). It can be observed that when $g_{2}$ increases, the fractional delay also increases as a result of the higher gain spectral curvature that reduces the effective full-width half-maximum gain bandwidth.
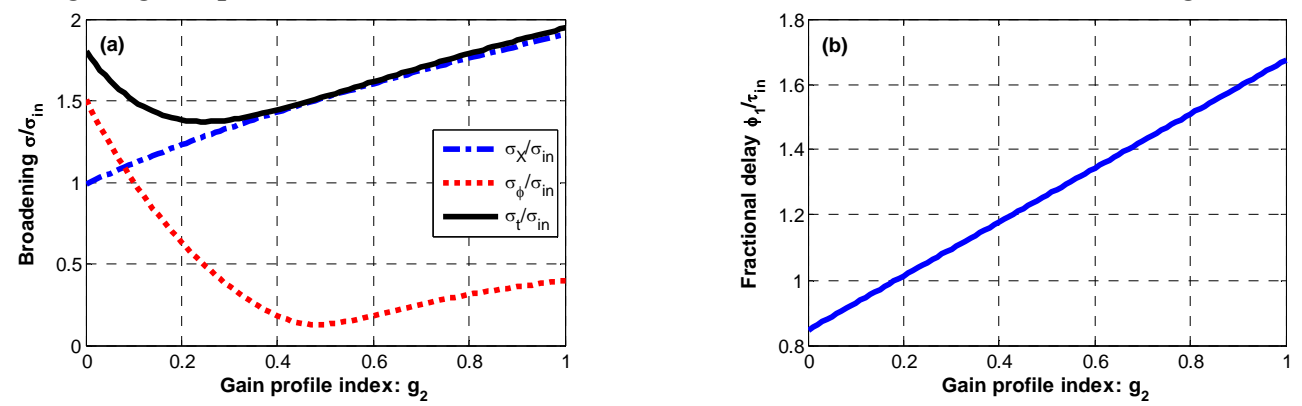

Fig. 1. (a) Pulse broadening (amplitude, phase and total contributions) and, (b) fractional delay as a function of the gain profile index $g_{2}$. The particular situation depicted here is for pulses of $880 \mathrm{ps}$ and a $32 \mathrm{~dB}$ peak SBS gain of $1 \mathrm{GHz}$ bandwidth.

\section{Experimental setup}

The experimental setup used to test these theoretical predictions is depicted in Fig. 2. The CW light from a distributed feedback (DFB) laser $(\lambda=1551 \mathrm{~nm})$ is divided into two branches by an optical coupler. The upper branch is used to generate the probe signal by using a Mach-Zehnder modulator (MZM) driven by a microwave source at a fixed frequency of $10.8 \mathrm{GHz}$ (corresponding to the peak Brillouin gain frequency in the fiber). A tunable fiber Bragg grating (FBG) is used to select one of the probe modulation sidebands. 880 ps probe pulses showing high extinction ratio are obtained by fast-switching a semiconductor optical amplifier (SOA), and then amplified using an erbiumdoped fiber amplifier (EDFA). A tunable optical filter (TOF) suppresses the EDFA out-of-band noise. The probe pulses are launched through an optical isolator into a $25 \mathrm{~km}$ standard single-mode fiber (SMF).

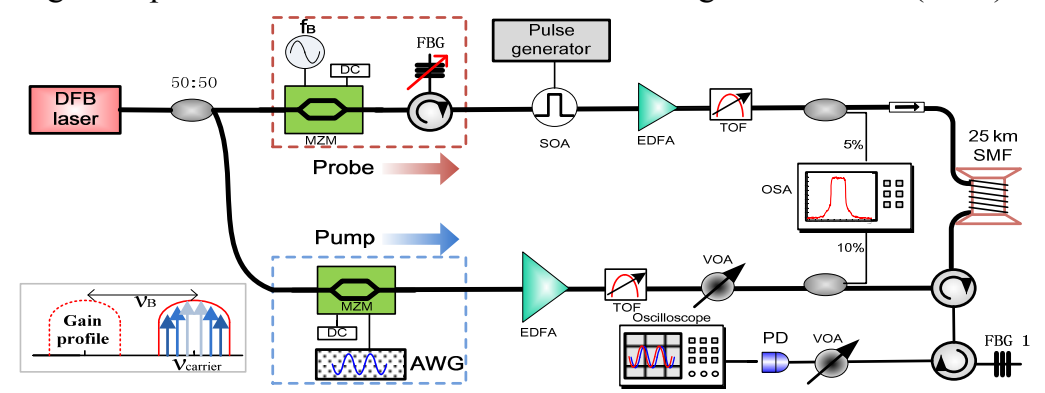

Fig. 2. Experimental setup for the measurement of fractional delay and pulse distortion using a gain-shaped SBS slow light medium.

Meanwhile, the light in the lower branch serves as the Brillouin pump wave and is modulated by a MZM operating in carrier-suppression mode using an arbitrary waveform generator (AWG). This way the optical spectrum of the pump can be precisely shaped through this external modulation with a frequency swept from 0 to $500 \mathrm{MHz}$ and a repetition period of $2 \mu \mathrm{s}$ (much smaller than the $125 \mu$ s propagation time along the $25 \mathrm{~km}$ SMF). Since both modulation sidebands are retained, a symmetric Brillouin gain spectrum spanning over $1 \mathrm{GHz}$ bandwidth is obtained. The so-tailored pump is amplified by an EDFA, its peak power is controlled by a variable optical attenuator (VOA), and then $10 \%$ of the power is monitored by an optical spectrum analyzer (OSA). Finally, the pump light is launched into the fiber through a circulator and utilized to amplify and delay the probe pulses. A circulator and a narrowband FBG are employed in the receiver to filter out the Rayleigh light backscattered from the pump. Pulses are then detected by a $1 \mathrm{GHz}$ photo-detector and a high-speed oscilloscope, after the adjustment of the peak power by a VOA.

\section{Results and concluding discussion}

Several gain profiles shaped under a generic quadratic form $g(\omega)=g_{0}-g_{2} \cdot(\omega / \Omega)^{2}$ over a strict $1 \mathrm{GHz}$ bandwidth have been generated by programing the AWG. The amplitude and phase responses of the SBS slow light medium have been simultaneously measured using a vector network analyzer (VNA), whose output electrical signal is used to modulate the probe with a frequency spanning the range $8-12 \mathrm{GHz}$, whilst the output (response) of the system is launched to a $25 \mathrm{GHz}$ detector connected to the input of the VNA. Fig. 3(a) shows that the flat-top gain profile (free from amplitude distortion) induces a strong phase distortion (highly non-linear phase response). The profile with $g_{2}=1$ in Fig. 3(c) introduces high amplitude distortion (strong low-pass effect) and moderate phase-distortion. The 
intermediate case with $g_{2}=0.5$ (Fig. 3(b)) shows a nearly perfect non-distorting linear phase response while keeping a limited low-pass effect on the amplitude. This is in excellent agreement with the expected theoretical results above described in Fig. 1.
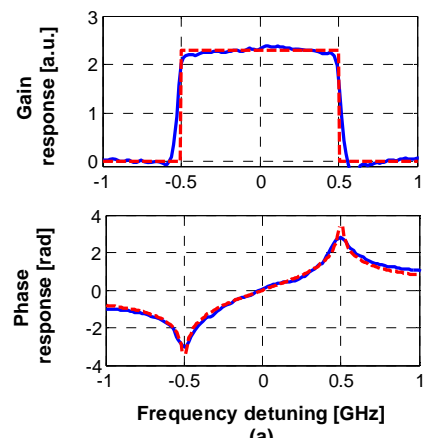

(a)
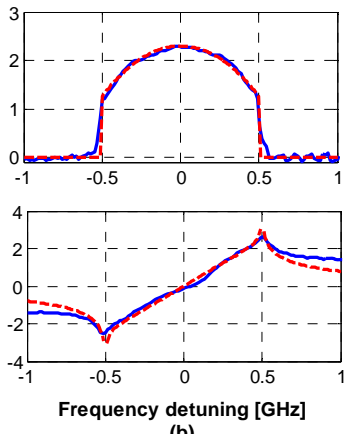

(b)
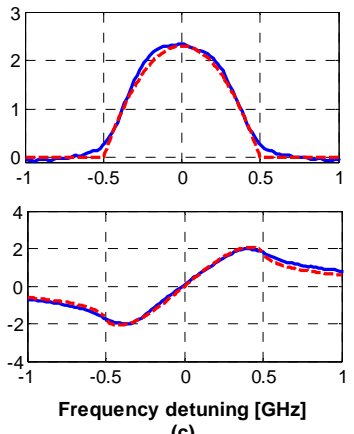

(c)

Fig. 3. Gain (top) and phase (bottom) response for three different gain profiles having $1 \mathrm{GHz}$ bandwidth and $20 \mathrm{~dB}$ gain $\left(g_{0}=2.3\right)$.

(a) $g_{2}=0$; (b) $g_{2}=0.5$; (c) $g_{2}=1$. Blue solid lines: measured gain profile and phase response. Red dashed lines: theoretical curves.

Shaped gain profiles are utilized for slow light generation to delay 880 ps pulses using the setup shown in Fig. 2 . Fig. 4(a) illustrates the time-domain waveforms of the delayed pulses for different gain profiles with $32 \mathrm{~dB}$ peak SBS gain. Enhanced delaying is found as expected by gradually changing the gain profile index $g_{2}$ from 0 up to 1 . A maximum delay of $1.27 \mathrm{~ns}$ is observed when $g_{2}=1$, while the flat-top gain profile with $g_{2}=0$ provides the minimum delay of $0.79 \mathrm{~ns}$, as expected from the theoretical analysis. A serious phase distortion can be found with indexes $g_{2}$ in the 0 to 0.25 range (evidenced by the second peak and long tails resulting from higher-order dispersion), whereas profiles with $g_{2}$ between 0.5 and 1 are dominated by amplitude distortion, showing a smooth low-pass filtering effect. These results fully agree with the theoretical predictions presented in Section 2.
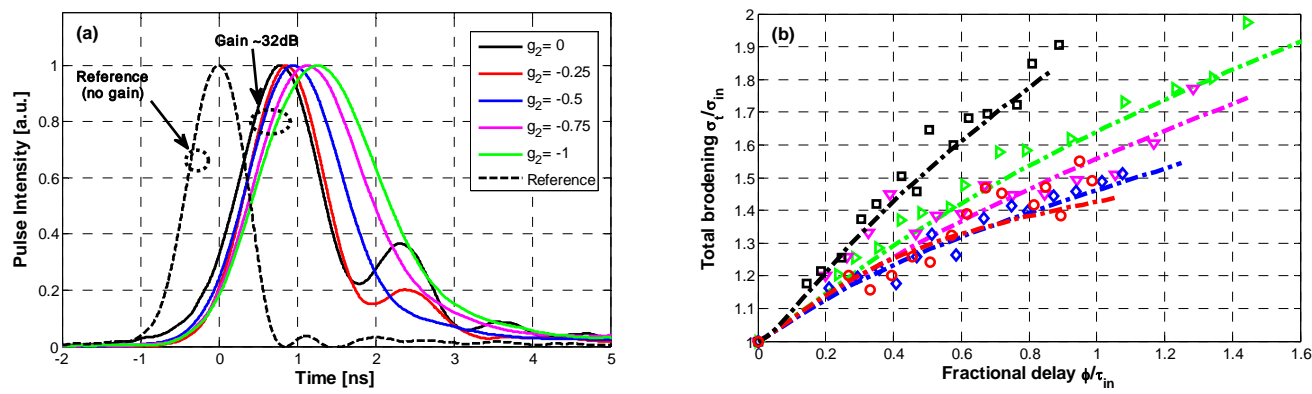

Fig. 4. (a) Normalized pulse waveforms of the reference with no gain (black dashed line) and of the delayed signals (colorful solid lines) for different gain profiles (with $32 \mathrm{~dB}$ gain). (b) Total broadening versus fractional delay (dots: experimental data; dashed lines: theoretical curves).

Finally, by calculating the RMS value of the delayed pulses, the experimental total broadening versus fractional delay is obtained for the different gain profiles, as depicted in Fig 4(b). This figure shows a good agreement between experiments (dots) and predictions (dashed lines), presented in Fig. 1 under the same conditions. Considering the tradeoff between amplitude-phase broadening and delaying power, optimal profiles are found for values of $g_{2}$ between 0.25 and 0.5 . Actually Fig. 4(b) clearly demonstrates that the flat-top gain profile (black dots/line) is not a good choice due to its poor delaying efficiency and the strong phase distortion observed at large fractional delays.

Even though distortion is known to be intimately bound to the delaying effect in a linear slow light system, it is shown here that there is some space for optimization and parabolic gain profiles with a profile index $g_{2}$ between 0.25 and 0.5 are proved here to minimize the pulse distortion for all usual fractional delays.

Liang Zhang acknowledges the support from the China Scholarship Council during his stay at EPFL.

\section{References}

[1] J. B. Khurgin and R. S. Taylor, Slow Light: Science and Applications (Taylor \& Francis, 2009).

[2] M. González-Herráez, S. Martin-Lopez, and L. Thévenaz, "Analytical expression of pulse broadening in an arbitrary linear slow light medium," Optics Letters 37, 3171-3173 (2012).

[3] R. Pant, M. Stenner, M. Neifeld, and D. Gauthier, "Optimal pump profile designs for broadband SBS slow-light systems," Opt. Express 16, 2764-2777 (2008).

[4] T. Sakamoto, T. Yamamoto, K. Shiraki, and T. Kurashima, "Low distortion slow light in flat Brillouin gain spectrum by using optical frequency comb," Opt. Express 16, 8026-8032 (2008). 